\title{
OHVIRA syndrome pre-operatively diagnosed using vaginoscopy and hysteroscopy: A case report
}

\author{
YASUSHI MABUCHI, JUNYA HIRAYAMA, NAMI OTA and KAZUHIKO INO
}

Department of Obstetrics and Gynecology, Wakayama Medical University, School of Medicine, Wakayama 641 0012, Japan

Received August 14, 2021; Accepted October 19, 2021

DOI: $10.3892 / \mathrm{mi} .2021 .20$

\begin{abstract}
Obstructed hemivagina and ipsilateral renal anomaly (OHVIRA) syndrome is a rare Mullerian duct anomaly. Cases with pelvic inflammatory disease or endometriosis have been reported, which may influence fertility or the quality of life of patients; therefore, an accurate and early diagnosis is essential. The present study reports the case of patient (25-year-old female, gravida 0 para 0 ) with OHVIRA syndrome. Magnetic resonance imaging and a computed tomography scan revealed uterus didelphys and right renal agenesis. An opening was found on the vaginal septum during the menstrual period. Upon diagnostic vaginoscopy and hysteroscopy through the opening, the right vaginal cavity was enclosed, and the right uterine cervix and cavity were normal. The patient was diagnosed with OHVIRA syndrome. Her vaginal septum was surgically removed under direct visualization and the pathological findings of the resected septum revealed a benign squamous epithelium. The post-operative course was uneventful and restenosis of the vagina was not observed. On the whole, the present study demonstrates that the technique, diagnostic vaginoscopy and hysteroscopy, is minimally invasive and is sufficient for the diagnosis of incomplete vaginal obstruction-type OHVIRA syndrome.
\end{abstract}

\section{Introduction}

Obstructed hemivagina and ipsilateral renal anomaly (OHVIRA) syndrome is a rare Mullerian duct anomaly. The exact incidence of OHVIRA syndrome is unknown. The major presentations are dysmenorrhea, lower abdominal pain, a paravaginal mass, abnormal vaginal discharge and intermenstrual bleeding (1).

In general, the majority of cases of OHVIRA syndrome are diagnosed during adolescence as the obstruction of menstrual outflow causes abdominal pain early following menarche.

Correspondence to: Dr Yasushi Mabuchi, Department of Obstetrics and Gynecology, Wakayama Medical University, School of Medicine, 8111 Kimiidera, Wakayama 641 0012, Japan

E-mail: booyan@wakayama-med.ac.jp

Key words: congenital abnormalities, endoscopy, hysteroscopy
Smith and Laufer (1) reported that the average age of diagnosis was 14 years. On the other hand, in the case of incomplete vaginal obstruction, the diagnosis is sometimes delayed due to the lack of symptoms. However, there are some cases of OHVIRA syndrome with pelvic inflammatory disease (2) or endometriosis (3), which may influence fertility or the quality of life of patients. Thus, it is crucial for an accurate diagnosis to be made and to provide appropriate treatment for patients with OHVIRA syndrome at an early age.

The present study reports a a case of a patient with OHVIRA syndrome who was admitted to hospital due to pelvic inflammatory disease. This diagnosed pre-operatively using vaginoscopy and hysteroscopy.

\section{Case report}

The present study describes the case of a 25-year-old female, gravida 0 para 0 , who visited an internal medicine clinic due to fever and lower abdominal pain. Her menarche occurred at age 11 and her menstrual cycle was regular. She regularly had sexual intercourse. She had no history of menorrhagia or dyspareunia. Magnetic resonance imaging (MRI) and a computed tomography (CT) scan revealed uterus didelphys and right renal agenesis (Fig. 1). She was referred to the authors' hospital as gynecological disease was suspected. Tenderness in the pouch of Douglas was noted upon a pelvic examination and C-reactive protein levels were found to be high upon a blood examination. The patient was diagnosed with pelvic inflammatory disease. The vaginal sidewall bulge was not detected by a speculum examination during the non-menstrual period. The right obstructed hemivagina was not clear upon the MRI examination. She was treated with antibiotics and recovered from pelvic inflammatory disease within a week. A few weeks later, an opening was found on the vaginal septum by a speculum examination during the menstrual period (Fig. 2A). Diagnostic vaginoscopy through the opening was performed using a flexible hysteroscope of $3.1 \mathrm{~mm}$ in diameter, which is usually used for diagnostic hysteroscopy in the outpatient clinic, without anesthetic agents. Upon diagnostic vaginoscopy and hysteroscopy, the right vaginal cavity was enclosed, and both the uterine cervix and uterine cavity were found to be normal (Fig. 2B and C). She was thus diagnosed with OHVIRA syndrome. Her vaginal septum was surgically removed under direct visualization and the pathological findings (hematoxylin and eosin staining, performed in the 

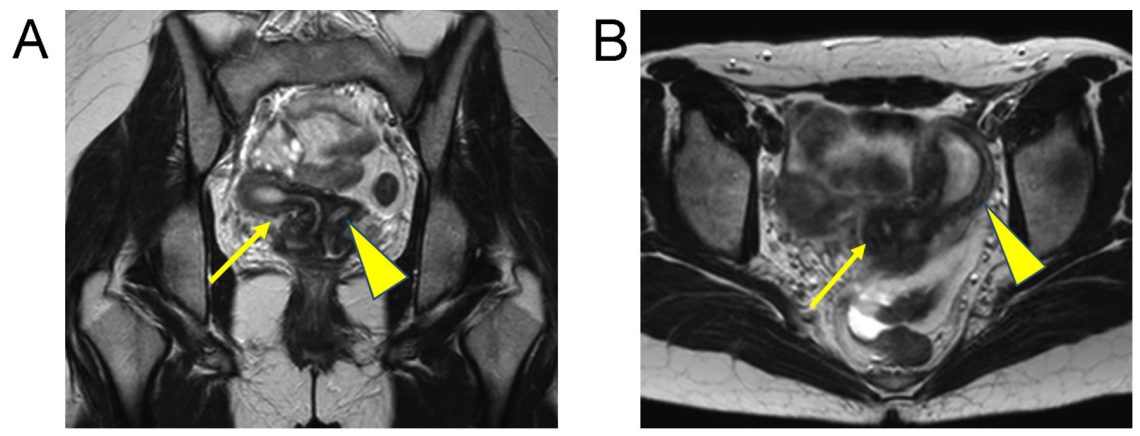

Figure 1. (A) Magnetic resonance image coronal view. (B) Magnetic resonance image axial view. Both images revealed uterus didelphys (the arrow indicates the right and the arrowhead indicates the left uterus). Magnetic resonance imaging did not reveal the communication site between the uteruses.

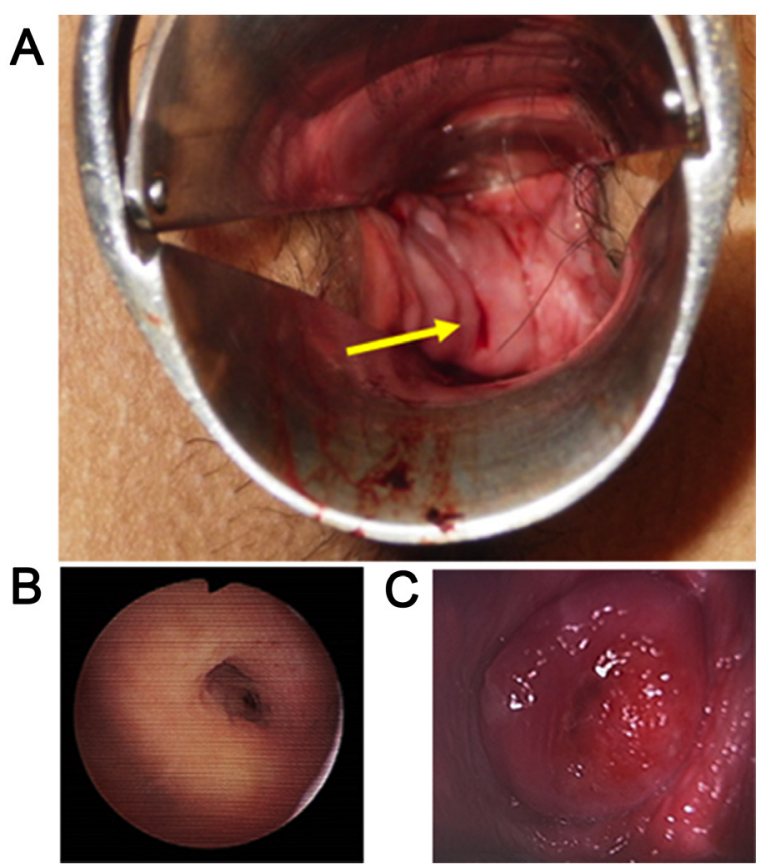

Figure 2. (A) Speculum examination during the menstrual period revealed an opening in the vaginal septum. The arrow indicates the opening in the vaginal septum. (B) Vaginoscopic view of the right cervix through the opening in the vaginal septum. (C) Vaginoscopic view of the left cervix.

A
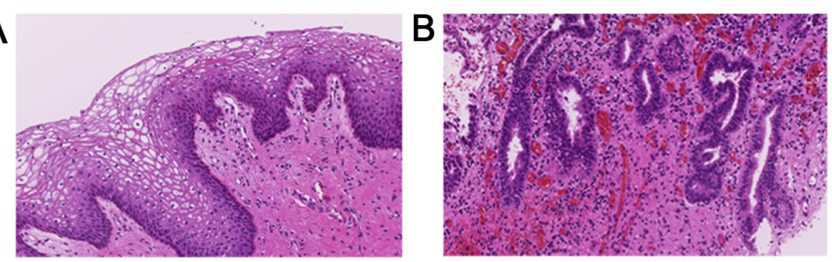

Figure 3. (A) Microscopic findings of the resected vaginal septum (hematoxylin and eosin staining; magnification, x200). Pathological findings of the resected septum revealed a benign squamous epithelium. (B) Vaginal adenosis was partially noted in the vaginal septum (hematoxylin and eosin staining; magnification, x200).

authors laboratory) of the resected septum revealed a benign squamous epithelium (Fig. 3A). The glandular epithelium was partially observed and vaginal adenosis was noted in the vaginal septum (Fig. 3B). The post-operative course was uneventful and restenosis of the vagina was not observed.

\section{Discussion}

OHVIRA syndrome is characterized by an obstructed hemivagina and ipsilateral renal anomaly. This syndrome was previously classified into three groups by Rock and Jones (4) as follows: Group 1, complete vaginal obstruction with a hematocolpos. Group 2, incomplete vaginal obstruction without a hematocolpos. In group 2, an opening in the partially obstructed vaginal pouch is observed. Group 3, complete vaginal obstruction with a laterally communicating double uterus. In the case presented herein, an opening was noted on the vaginal septum and the communication between the uteruses could not be observed. Thus, the patient was diagnosed with OHVIRA syndrome with group 2 classification. Smith and Laufer (1) reported 27 cases of OHVIRA syndrome. The mean age at diagnosis was 14 years. A total of 23 patients had ipsilateral renal anomalies, including 20 with renal agenesis. In addition, 26 patients underwent vaginal reconstruction, eight of whom additionally underwent laparoscopy for the classification of the diagnosis. Furthermore, 6 patients required two-stage vaginoplasty due to incomplete previous resection $(n=1)$, infection or anatomic distortion $(n=4)$, or restenosis $(n=2)$. Vaginal septum adenosis was noted in 7 patients. In their study, they concluded that the majority of patients with OHVIRA syndrome can be treated solely by single- stage vaginoplasty and that routine laparoscopy is not essential for management. They also described two cases of post-operative restenosis among 7 patients with vaginal adenosis in the resected vaginal septum (1). Candiani et al (3) reported 36 cases of double uterus, blind hemivagina and ipsilateral renal agenesis in 1997. In their study, the vaginal septum was excised and marsupialization was carried out in 30 out of the 36 cases. The pregnancy rate among the 15 women wanting children was $87 \%$ and the live birth rate was $77 \%$ (3). Kamio et al (2) reported a case of OHVIRA syndrome with septic shock. In that case, a 37-year-old female was diagnosed with OHVIRA syndrome and she developed septic shock due to pyocolpos, requiring intensive care unit hospitalization (2). According to another study, patients with OHVIRA syndrome can develop pyocolpos, abscesses or pelvic inflammatory disease (5).

As aforementioned, the majority of cases of OHVIRA syndrome are diagnosed during adolescence as the obstruction of menstrual outflow causes abdominal pain early following menarche. On the other hand, in the case of incomplete vaginal 
obstruction, such as in the case in the present study, the diagnosis is sometimes delayed due to the lack of symptoms. However, there are some cases of OHVIRA syndrome with pelvic inflammatory disease (2) or endometriosis (3), which may influence fertility or the quality of life of patients. Thus, it is important to make an accurate diagnosis and provide appropriate treatment for patients with OHVIRA syndrome at an early age.

In the present case, the patient did not exhibit menorrhalgia or dyspareunia, although she regularly had sexual intercourse. Due to the lack of symptoms related to menstruation or coitus, she had not visited a gynecologist until the occurrence of pelvic inflammatory disease. She visited an internal medicine clinic due to experiencing fever and lower abdominal pain, and the judgment by the physician led to a correct diagnosis of OHVIRA syndrome. It was not difficult to diagnose uterus didelphys and right renal agenesis by MRI and a CT scan. An opening was found on the vaginal septum by speculum examination during the menstruation period. Diagnostic vaginoscopy through the opening was performed without the leakage of saline solution as the opening and the fiberscope had almost same diameter. Diagnostic vaginoscopy and hysteroscopy through the opening were also useful to observe the obstructed vaginal cavity and the right uterine cavity. In the case presented herein, pelvic inflammatory disease was considered to be associated with OHVIRA syndrome due to the stasis of menstrual blood and regular sexual intercourse. Her vaginal septum was surgically removed and the pathological findings of the resected septum revealed benign squamous and glandular epithelium. Thus, vaginal adenosis was noted in the vaginal septum and attention should be paid to vaginal restenosis, as previously reported (1). Following surgery, the reduction of the risk of pelvic inflammatory disease and normal childbearing is expected.

In the cases of uterus didelphys without hematocolpos, such as in the case presented herein, a speculum examination during the menstruation period is useful for searching the menstrual outflow passage. If a passage is not found in the vagina, an exploratory laparoscopy or laparotomy is then required to identify the communication locus between the uteruses. These procedures are crucial in order to avoid misdiagnoses in the cases of incomplete vaginal obstruction without hematocolpos.

Cheng et al (6) reported the technique of vaginoscopic incision of the vaginal septum in patients with OHVIRA syndrome. They described that vaginoplasty can be performed under direct visualization with a speculum for adult patients; however, vaginoscopic management using the non-touch technique may be an option for adolescent patients with an immature and narrow vagina (6). Johary et al (7) reported the use of a hysteroscope for diagnostic vaginoscopy and/or hysteroscopy in their systematic review. Speculum inspection has limited ability to observe the entire vagina, and may cause pain and increase the risk of hymen injury in patients who are virgins. Johary et al (7) reported that diagnostic vaginoscopy using flexible hysteroscope may minimize the risk of hymen injury and may serve as an alternative option for adolescent patients. Shih et al (8) reported the use of a flexible hysteroscope for the diagnosis of uterus didelphys, a vertical vaginal septum and an obstructed hemivagina with pyocolpos in a 15-year-old patient. In the case in the present study, the patient was 25 years of age and had regular sexual intercourse. Diagnostic vaginoscopy through the opening on the vaginal septum for the purpose of hemivagina cavity observation differed from the aforementioned report about diagnostic vaginoscopy in an adolescent. To date, to the best of our knowledge, there are no studies available on diagnostic vaginoscopy through the opening on the vaginal septum in incomplete vaginal obstruction-type OHVIRA syndrome.

In conclusion, the case described in the present study was pre-operatively diagnosed with OHVIRA syndrome by vaginoscopy and hysteroscopy. A speculum examination during the menstrual period revealed the outflow of menstrual blood through the opening on the vaginal septum. This technique, diagnostic vaginoscopy and hysteroscopy, is minimally invasive and sufficient for the diagnosis of incomplete vaginal obstruction-type OHVIRA syndrome. An accurate pre-operative diagnosis enabled less-invasive surgical treatment solely by the resection of the vaginal septum, avoiding exploratory laparoscopy or laparotomy.

\section{Acknowledgements}

Not applicable.

\section{Funding}

No funding was received.

\section{Availability of data and materials}

The dataset used and/ or analyzed during the current study are available from the corresponding author on reasonable request.

\section{Authors' contributions}

All authors (YM, JH, NO and KI) participated in the conception and design of the study. YM, JH and NO obtained the data and treated the patient. YM examined the patient data and drafted the manuscript. KI revised the manuscript prior to submission. In addition, KI and YM were major contributors to the design of the study. YM and KI confirm the authenticity of all the raw data. All authors have read and approved the final version of the manuscript.

\section{Ethics approval and consent to participate}

The patient provided written informed consent for the presentation of her case.

\section{Patient consent for publication}

The patient provided written informed consent for the publication of any associated data and accompanying images.

\section{Competing interests}

The authors declare that they have no competing interests. 


\section{References}

1. Smith NA and Laufer MR: Obstructed hemivagina and ipsilateral renal anomaly (OHVIRA) syndrome: Management and follow-up. Fertil Steril 87: 918-922, 2007.

2. Kamio M, Nagata C, Sameshima H, Togami S and Kobayashi $H$ : Obstructed hemivagina and ipsilateral renal anomaly (OHVIRA) syndrome with septic shock: A case report. J Obstet Gynaecol Res 44: 1326-1329, 2018

3. Candiani GB, Fedele L and Candiani M: Double uterus, blind hemivagina, and ipsilateral renal agenesis: 36 cases and long-term follow-up. Obstet Gynecol 90: 26-32, 1997.

4. Rock JA and Jones HW Jr: The double uterus associated with an obstructed hemivagina and ipsilateral renal agenesis. Am J Obstet Gynecol 138: 339-342, 1980.

5. Zurawin RK, Dietrich JE, Heard MJ and Edwards CL: Didelphic uterus and obstructed hemivagina with renal agenesis: Case report and review of the literature. J Pediatr Adolesc Gynecol 17: 137-141, 2004.
6. Cheng C, Subedi J, Zhang A, Johnson G, Zhao X, Xu D and Guan X: Vaginoscopic incision of oblique vaginal septum in adolescents with OHVIRA syndrome. Sci Rep 9: 20042, 2019.

7. Johary J, Xue M, Xu B, Xu D and Aili A: Use of hysteroscope for vaginoscopy or hysteroscopy in adolescents for the diagnosis and therapeutic management of gynecologic disorders: A systematic review. J Pediatr Adolesc Gynecol 28: 29-37, 2015.

8. Shih CL, Hung YC, Chen CP, Chien SC and Lin WC: Resectoscopic excision of the vaginal septum in a virgin with uterus didelphys and obstructed unilateral vagina. Taiwan J Obstet Gynecol 49: 109-111, 2010.

This work is licensed under a Creative Commons Attribution-NonCommercial-NoDerivatives 4.0 International (CC BY-NC-ND 4.0) License. 